\title{
Protocol for a randomized controlled trial examining multilevel prediction of response to behavioral activation and exposure-based therapy for generalized anxiety disorder
}

\author{
J. Santiago', E. Akeman', N. Kirlic ${ }^{1}$, A. N. Clausen ${ }^{4,5}$, K. T. Cosgrove ${ }^{1,3}$, T. J. McDermott ${ }^{1,3}$, B. Mathis ${ }^{1}$, M. Paulus ${ }^{1,2}$, \\ M. G. Craske ${ }^{6}$, J. Abelson ${ }^{7}$, C. Martell ${ }^{8}$, K. Wolitzky-Taylor ${ }^{6}$, J. Bodurka ${ }^{1,9}$, W. K. Thompson ${ }^{10}$ and Robin L. Aupperle ${ }^{1,2^{*}}$ (D)
}

\begin{abstract}
Background: Only 40-60\% of patients with generalized anxiety disorder experience long-lasting improvement with gold standard psychosocial interventions. Identifying neurobehavioral factors that predict treatment success might provide specific targets for more individualized interventions, fostering more optimal outcomes and bringing us closer to the goal of "personalized medicine." Research suggests that reward and threat processing (approach/ avoidance behavior) and cognitive control may be important for understanding anxiety and comorbid depressive disorders and may have relevance to treatment outcomes. This study was designed to determine whether approach-avoidance behaviors and associated neural responses moderate treatment response to exposure-based versus behavioral activation therapy for generalized anxiety disorder.
\end{abstract}

Methods/design: We are conducting a randomized controlled trial involving two 10-week group-based interventions: exposure-based therapy or behavioral activation therapy. These interventions focus on specific and unique aspects of threat and reward processing, respectively. Prior to and after treatment, participants are interviewed and undergo behavioral, biomarker, and neuroimaging assessments, with a focus on approach and avoidance processing and decision-making. Primary analyses will use mixed models to examine whether hypothesized approach, avoidance, and conflict arbitration behaviors and associated neural responses at baseline moderate symptom change with treatment, as assessed using the Generalized Anxiety Disorder-7 item scale. Exploratory analyses will examine additional potential treatment moderators and use data reduction and machine learning methods.

Discussion: This protocol provides a framework for how studies may be designed to move the field toward neuroscience-informed and personalized psychosocial treatments. The results of this trial will have implications for approach-avoidance processing in generalized anxiety disorder, relationships between levels of analysis (i.e., behavioral, neural), and predictors of behavioral therapy outcome.

Trial registration: The study was retrospectively registered within 21 days of first participant enrollment in accordance with FDAAA 801 with ClinicalTrials.gov, NCT02807480. Registered on June 21, 2016, before results.

Keywords: Generalized anxiety disorder, Depression, Behavioral activation, Exposure therapy, Cognitive behavioral therapy, Functional magnetic resonance imaging

\footnotetext{
* Correspondence: raupperle@laureateinstitute.org

${ }^{1}$ Laureate Institute for Brain Research, 6655 South Yale Avenue, Tulsa, OK

74136, USA

${ }^{2}$ School of Community Medicine, University of Tulsa, Tulsa, OK, USA

Full list of author information is available at the end of the article
}

(c) The Author(s). 2020 Open Access This article is distributed under the terms of the Creative Commons Attribution 4.0 International License (http://creativecommons.org/licenses/by/4.0/), which permits unrestricted use, distribution, and reproduction in any medium, provided you give appropriate credit to the original author(s) and the source, provide a link to the Creative Commons license, and indicate if changes were made. The Creative Commons Public Domain Dedication waiver (http://creativecommons.org/publicdomain/zero/1.0/) applies to the data made available in this article, unless otherwise stated. 


\section{Background}

Anxiety disorders are the most common mental health problem in the United States [1], and generalized anxiety disorder (GAD) is the most common anxiety disorder in primary care, with a lifetime prevalence rate of $6 \%$ [2]. It is a debilitating disorder leading to significant individual and socioeconomic burden with estimated annual costs of over $\$ 1500$ per patient $[2,3]$. Its prognosis is poor, with only $58 \%$ of cases experiencing remission within 2 years [4]. $\mathrm{GAD}$ is accompanied by major depressive disorder (MDD) in approximately $72 \%$ of cases, while MDD is accompanied by GAD in $48 \%$ of cases [5]. GAD in those with depression predicts poorer clinical outcomes and increased suicidal ideation compared with those with depression alone [6].

Psychotropic medications (e.g., selective serotonin reuptake inhibitors [SSRIs]) and psychotherapeutic interventions (e.g., cognitive behavioral therapy [CBT]) are both effective evidence-based treatments for GAD [7, 8]. However, only $40-60 \%$ of patients experience improvement with these treatments $[9,10]$, and $15-25 \%$ of those who improve relapse within 1 year [9]. This creates both clinical and socioeconomic challenges because these treatments are costly and time-consuming [11]. By identifying cognitive, behavioral, or neural factors that predict outcomes and can perhaps be targeted in an individualized fashion, we can move toward personalized approaches that assign each patient to the optimal treatment for them.

The National Institute of Mental Health (NIMH) Research Domain Criteria (RDoC) initiative seeks to improve mental health assessment and treatment by enhancing understanding of basic psychological domains across multiple levels of analysis (e.g., neural systems, physiology, behavior) [12]. Ideally, enhanced understanding of these domains will inform personalized treatment approaches. For example, if individual profiles of functioning across positive valence (e.g., approaching reward), negative valence (e.g., avoiding threat), or cognitive function (e.g., cognitive control) domains are identifiable, they could predict likelihood of success for various treatment approaches [13]. To achieve this eventual goal of "personalized medicine" [14], it is necessary to conduct clinical trials assessing these multilevel domains of function, randomize participants to comparator treatments, and examine common and unique predictors of treatment outcome.

Symptom severity, chronicity of symptoms, and comorbidity have been identified as potential predictors of GAD treatment response [15], but these findings do not provide specific targets for improving treatment effectiveness. The neurocognitive investigation of GAD has focused on enhanced negative affect and threat detection, as well as contradictory theories of either inadequate top-down prefrontal cortex (PFC) regulation (e.g., of amygdala) or PFC overactivation supporting maladaptive cognitive strategies (e.g., worry) [16]. There have been few neuroimaging studies examining predictors of psychosocial treatment response with GAD [17]. One study indicated that greater frontal, temporal, and insular activation during emotion reappraisal may predict better CBT response [18], but no GAD study to date has examined the use of neuroimaging to uniquely predict outcomes of two effective but divergent interventions.

Anxiety disorders have been hypothesized to arise from conflicting motivations to approach or avoid anxiogenic situations that also contain potential gains, leading to chronic distress, uncertainty, and use of maladaptive coping mechanisms (i.e., avoidance, worry) $[19,20]$. Animal paradigms thought to be relevant for GAD rely heavily on approach-avoidance conflict (AAC) (such as Vogel or Geller-Seifter conflict test, in which a behavior is associated with both rewards, e.g., food pellet, and punishment, e.g., shock) [21, 22]. We developed a human AAC task [23, 24] for use in functional magnetic resonance imaging (fMRI) work, and we have shown that approach behavior was linked to caudate and anterior cingulate cortex activation, whereas difficulties arbitrating conflict were linked to self-reported anxiety and dorsolateral prefrontal cortex (dlPFC) activation. Given that GAD treatments typically focus on decreasing cognitive and behavioral avoidance [25], the ability to successfully arbitrate conflict (make decisions to approach or avoid) could theoretically contribute to propensity for treatment response. MDD has been associated with dysfunction in both reward and threat processing [26], and MDD treatments often focus on increasing meaningful engagement in rewarding or pleasurable activities [27]. Thus, approach-avoidance processing is likely important in understanding treatment for the clinical presentation of GAD with or without comorbid depression.

Herein we present the protocol of an ongoing study designed to address the need for identifying moderators of GAD treatment response. This protocol explores multilevel moderators (self-report, behavioral, and neuroimaging) of response to exposure therapy (EXP) [25, 28 ] versus behavioral activation (BA) [29]. These interventions were chosen due to their (1) likelihood of being effective for GAD and (2) specific and unique functional targets relating to RDoC domains (i.e., avoidance/threat targeted by EXP versus approach/ reinforcement processing targeted by BA; see Fig. 1). We focused on the following aims:

1. Examine relationships among multilevel approachavoidance behavior and neural responses and baseline GAD symptom severity

2. Examine how multilevel approach-avoidance behavior and neural responses moderate individualized response to exposure-based therapy versus BA for GAD 
3. Identify the changes in approach-avoidance processes that relate to EXP- versus BA-elicited symptom improvement

\section{Methods/design}

This protocol was written using the Standard Protocol Items: Recommendations for Interventional Trials (SPIRIT) guidelines, and the SPIRIT checklist is provided in Additional file 2. The protocol is part of an ongoing, randomized (two-condition), single-center (Laureate Institute for Brain Research [LIBR], Tulsa, OK, USA), controlled trial examining multilevel predictors of response to EXP versus $\mathrm{BA}$ for GAD. The study is currently recruiting and is registered with ClinicalTrials.gov (identifier NCT02807480; registration date June 21, 2016). No amendments have been made to the protocol since original submission to ClinicalTrials.gov. The study is funded by the National Institute of Mental Health (grant K23MH108707; Robin L. Aupperle [RLA], principal investigator [PI]) and the William K. Warren Foundation. Interventions include 10 weeks of manualized, group-based BA or EXP therapy. Groups of 8-10 participants are randomized altogether to a therapy group (randomization conducted in blocks of 4; sequence generated by RLA). Participants are kept blind to their intervention condition until completion of all baseline assessments; outcome assessors are partially blinded (see further description in Additional file 1). Primary predictor variables of interest are assessed using the approach-avoidance task (AAT) and the AAC task, whereas the primary outcome measure is the GAD-7. Secondary outcome measures include the Sheehan Disability Scale [30], NIH Patient-Reported Outcomes Measurement Information System anxiety and depression scales [31], Beck Depression Inventory-II (BDI-II) [32], and Penn State Worry Questionnaire [33].

The overall study protocol is presented in Fig. 2. Screening assessments confirm exclusion and inclusion criteria for the study; baseline assessment includes self-report, behavioral, biological, and neuroimaging assessments. After baseline assessment, individuals are randomized to

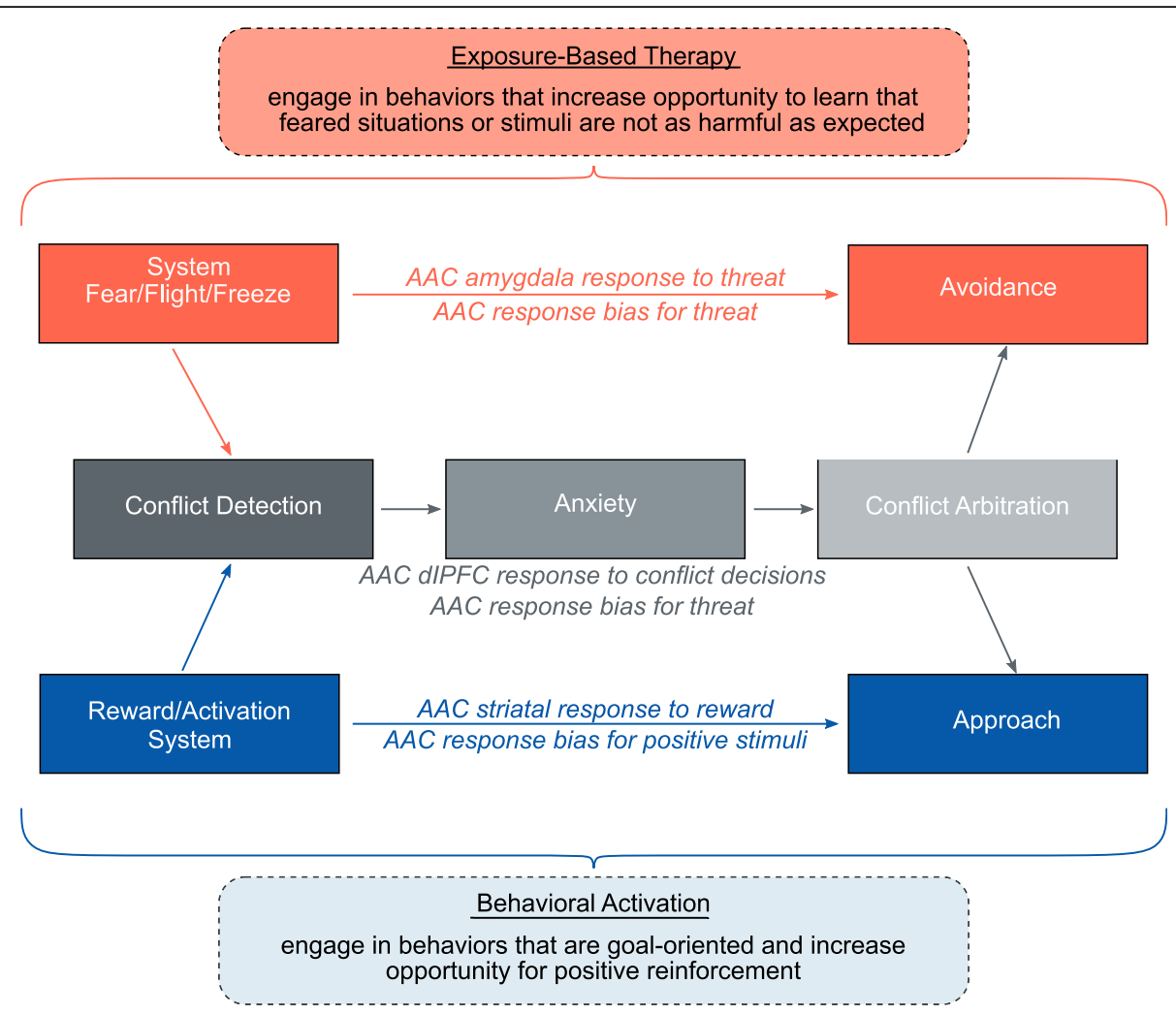

Fig. 1 Approach-avoidance conflict model that provided the bases for the current study protocol, aims, and hypotheses. As illustrated, both the fear or avoidance system and the reward/approach system are considered important in eliciting conflict and anxiety. Conflict arbitration requires appropriate balancing of both approach and avoidance drives. In the current protocol, approach and avoidance behaviors are defined by approach-avoidance test (AAT) bias scores; conflict arbitration is defined by reaction time during the approach-avoidance conflict (AAC) trials. For brain responses, we focus on the AAC task and extract percentage signal change (PSC) from a priori regions of interest: (1) approach: left caudate (reward versus no-reward outcome), (2) avoidance: right amygdala (negative versus positive affective outcome), and (3) conflict: right dorsolateral prefrontal cortex (dIPFC; conflict versus nonconflict decisions). Exposure-based therapy was included as a treatment that primarily targets avoidance or threat processes, whereas behavioral activation was included as a treatment that primarily targets approach or reward systems 
EXP or BA treatment, during which weekly self-report symptom measures are obtained. After treatment, participants repeat baseline assessments. Self-report symptom measures are repeated at 3 and 6 months following treatment. Research is conducted ethically in accordance with the World Medical Association Declaration of Helsinki. Research personnel trained in human subject research obtain written informed consent from each participant prior to completing any research procedures. The consent form for the study is included in Additional file 5 .

\section{Participants}

Projected enrollment is 100 treatment-seeking individuals meeting Diagnostic and Statistical Manual of Mental Disorders, Fifth Edition (DSM-5), GAD criteria [34] over a 5year period (April 2016-April 2021), recruited from community mental health clinics and the general community through electronic and print advertisements. Participants must be 18-55 years old, have sufficient English proficiency to understand study procedures, and meet DSM-5 criteria per the Mini International Neuropsychiatric Interview (MINI 7.0) for GAD and score $>7$ on the Overall Anxiety Severity and Impairment Scale [35]. Participants are excluded for the following reasons: (1) severe depressive symptoms (Patient Health Questionnaire-9 score > 17) and/ or suicidal ideation with intent or plan, to decrease safety concerns and help ensure that GAD was the primary cause of impairment; (2) history of substance use disorder in the past 6 months; (3) meeting diagnostic criteria for psychotic, bipolar, obsessive-compulsive, or eating disorders; (4) moderate to severe traumatic brain injury or other neurocognitive disorder; (5) severe or unstable medical conditions, (6) magnetic resonance imaging (MRI) contraindications, such as metal or metallic devices in the body; (7) noncorrectable vision or hearing problems; and (8) current use of psychotropic medications that could affect brain function (e.g., anxiolytics, antipsychotics, or mood stabilizers). Participants reporting current use of antidepressants (SSRIs) are included as long as the dose has been stable for 6 weeks prior to enrollment. Inclusion/exclusion criteria are meant to decrease potential confounders while also supporting generalizability of results to GAD patient populations in the community.

\section{Intervention}

Both BA and EXP treatments consist of manualized, tensession interventions and are delivered in a group format for 90 min per week. For each group, participants are provided a binder to accompany the intervention, including outlines of each session, basic descriptions of concepts, and "homework" worksheets. Brief descriptions of each intervention are provided below and in Table 1. Descriptions of treatment compliance assessments and strategies are provided in Additional file 1.

\section{$B A$}

BA is a recognized efficacious treatment for MDD [36] and is based on the premise that negative or stressful life

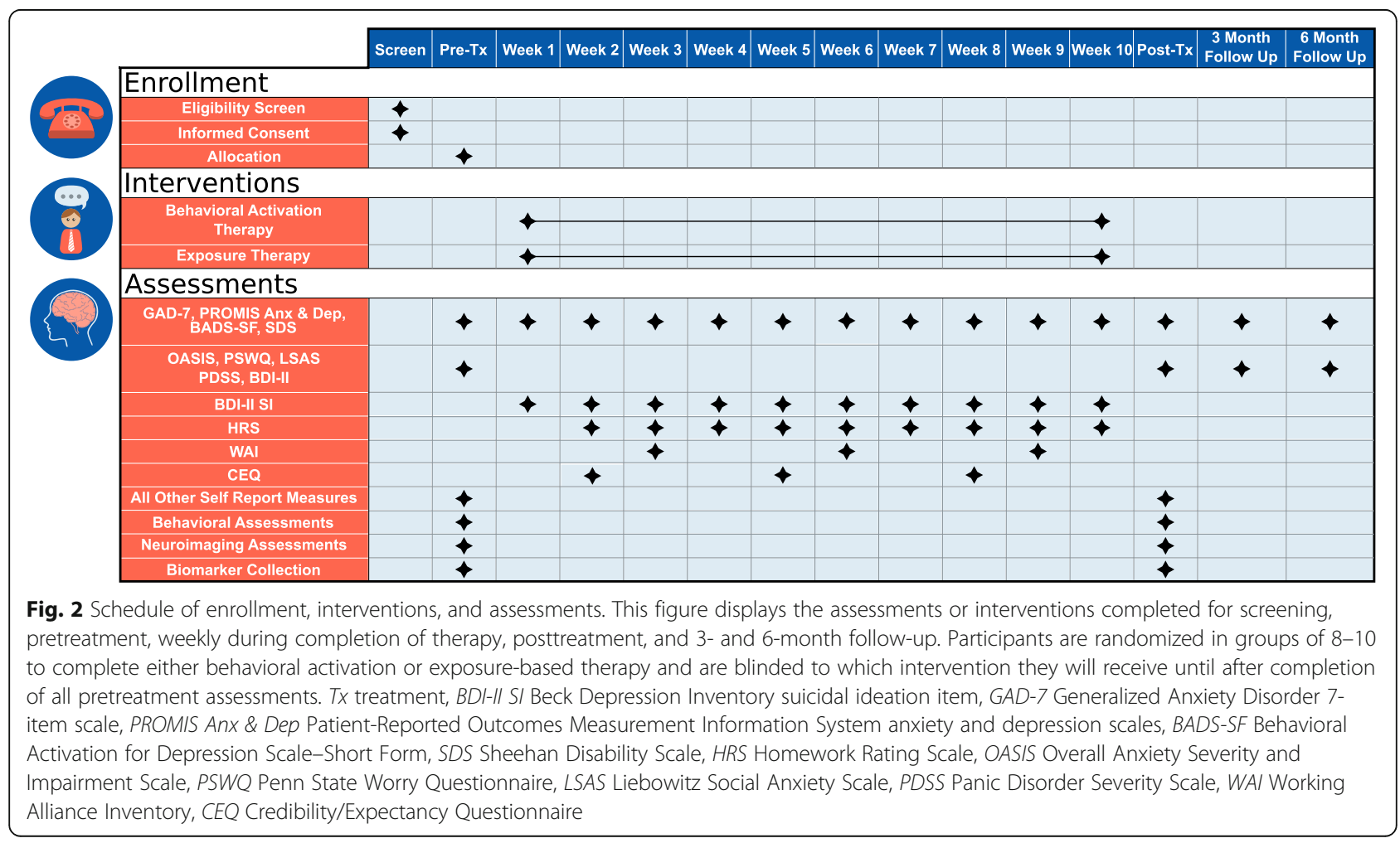




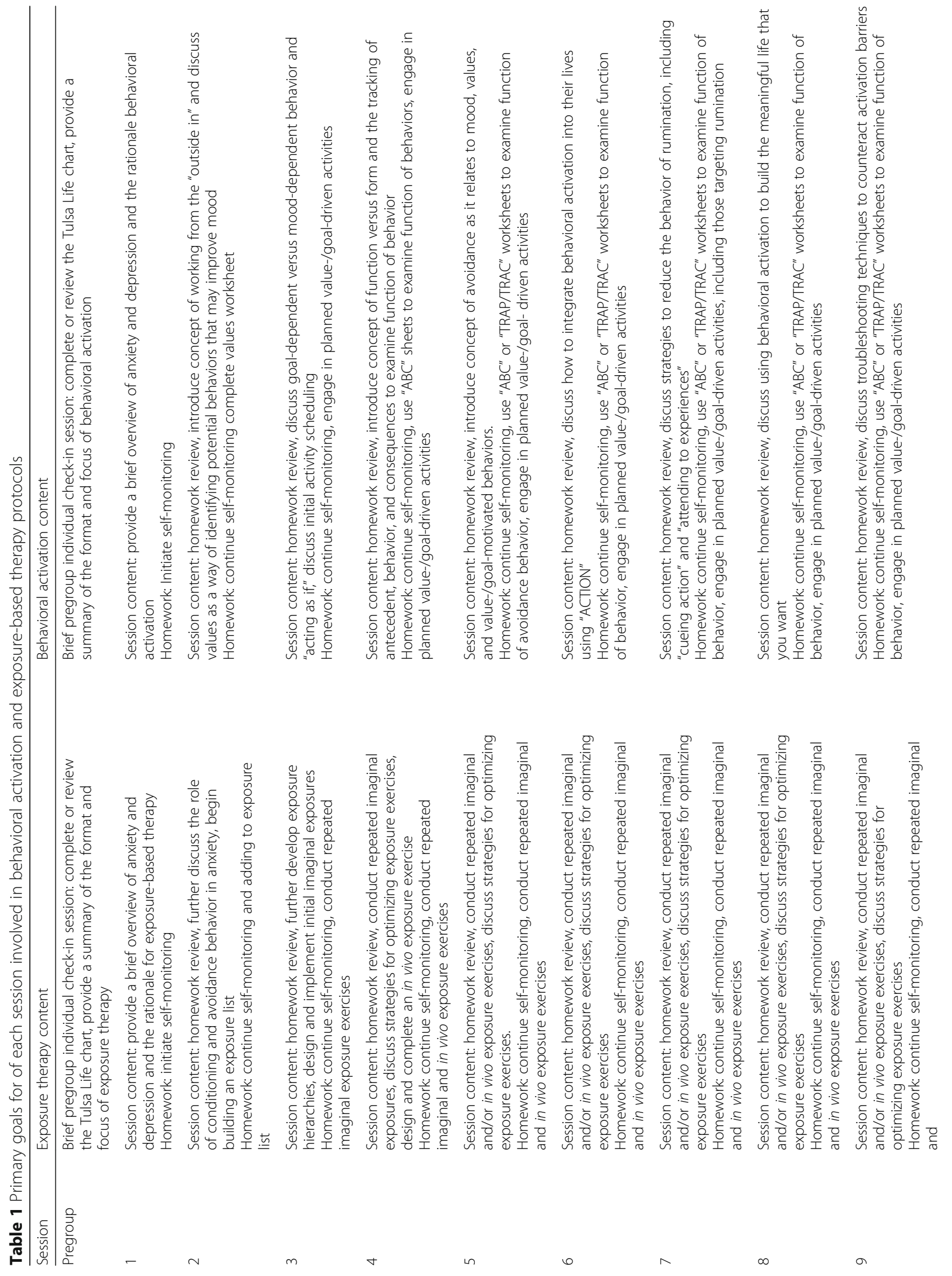




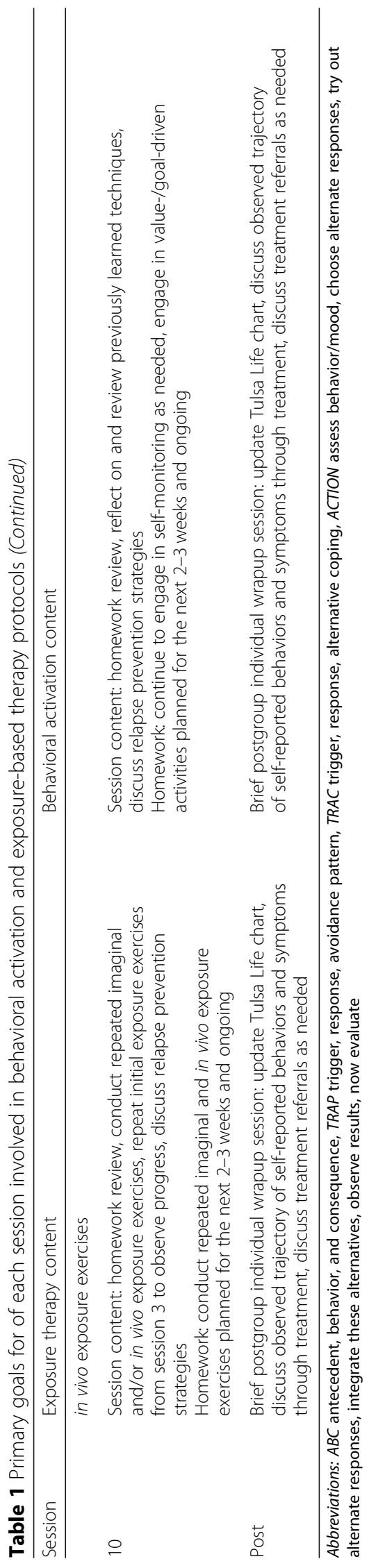


events can reduce one's ability to experience reward or reinforcement (e.g., reduced social support). Depression develops and is maintained when individuals respond in ways that create additional deficits in reward or reinforcement (e.g., further isolation). The goal of BA is to identify alternative behaviors to increase in a way that increases opportunities for reward or reinforcement, particularly through naturally reinforcing behaviors (e.g., those related to one's values). A ten-session, structured, group-based BA manual was developed by coauthors RLA and CM (with edits and revisions provided by AC), informed by previously published BA treatment guides [29] and modified to focus on negative mood more generally rather than solely on depression.

\section{EXP}

EXP is a recognized efficacious strategy for the treatment of anxiety disorders. EXP is based on the premise that anxiety arises from a perceived threat associated with discrete cues or contexts, whether from direct or indirect/vicarious experience or informational transmission of perceived threat. Anxiety is thought to be maintained by avoidance behavior, preventing corrective learning. EXP guides individuals to decrease avoidance and experience anxiety-provoking situations or cues in a safe environment, allowing for inhibitory learning or habituation. The ten-session, structured, groupbased EXP manual was based on a previous group-based anxiety treatment manual [37] developed by MGC, modified further by MGC and RLA (with edits and revisions provided by KWT and AC), to focus on exposure strategies only (without cognitive restructuring) and inhibitory learning rather than habituation only [38].

\section{Therapist training and treatment fidelity}

Each EXP and BA group intervention is delivered by two cotherapists: a licensed doctoral- or master's-level clinician with either another licensed clinician or a therapist in training (i.e., clinical psychology postdoctoral fellow or graduate student). Each therapist completes in-person or online workshops (e.g., Behavioral Tech, LLC, https:// behavioraltech.org; Centre for Research on Eating Disorders at Oxford, https://credo-oxford.com), reads articles and manuals related to each treatment [29, 38], and watches videos of previous therapy sessions. Each therapy session is video and audio recorded, and at least $20 \%$ of sessions will be randomly selected for fidelity ratings. Skill acquisition and fidelity are assessed using the Quality of Behavioral Activation Scale for BA (Dimidjian, Hubley, Martell, Herman-Dunn, and Dobson, 2012, unpublished measure) and a fidelity form created for the EXP treatment by RLA in consultation with MGC and KWT. Fidelity ratings will be provided by experts in each therapy (KWT and $\mathrm{CM}$ ) or their trainees. Each therapist attends weekly consultation and supervision with the PI and/or consultants.

\section{Data collection}

All interview-based assessments (e.g., MINI) are administered by experienced, blinded examiners trained to high levels of interrater reliability (kappa $>0.80$ ). Self-report data are collected electronically using Research Electronic Data Capture (REDCap) [39]. Study consent records are stored in a locked records room at LIBR. Study data records and blood/urine/biological samples are assigned code numbers and are not individually identifiable. REDCap servers are housed in a local data center at LIBR, and all web-based information transmission is encrypted.

\section{Measures}

Self-report, behavioral, and neuroimaging measures included in the protocol are listed in Table 2 (refer to Fig. 2 for timing of measures). Below are descriptions of the behavioral and neuroimaging tasks serving as primary predictors of interest. The remaining tasks are described in Additional file 1.

\section{AAT}

The AAT assesses behavioral avoidance tendencies [71]. Participants are shown a picture of an emotional face (happy, angry, or neutral) framed by a blue or yellow border and instructed to pull a joystick (approach) when the border is one color and to push it away (avoid) when it is the other (counterbalanced). The picture zooms out and in accordingly. Mean response latency for push is subtracted from pull (e.g., angry pull - angry push) to obtain an avoidance bias score.

\section{AAC task}

The AAC task probes decision-making processes during approach-avoidance conflict [23, 24] (Fig. 3). On each trial, the subject decides between two outcomes, which are represented on each side of a runway. A cloud indicates that a negative affective image/sound pair will be shown, and a sun indicates that a positive image/sound pair will be shown (e.g., from International Affective Picture System and International Affective Digitized Sounds System [72, 73]). The amount of red in a rectangle indicates the amount of money awarded for each option $(2,4$, or 6 cents). For conflict trials, negative stimuli are paired with rewards. Thus, the same behavior leads to both affective punishment and reward. For nonconflict "approach" trials, both outcomes include positive affective stimuli, but only one offers a reward. For "avoid" trials, neither outcome offers a reward, but one involves a negative affective image. For each trial, the subject moves the avatar, knowing that the probability of each outcome (10$90 \%)$ depends on their end position. Behavioral variables include approach behavior (end avatar position) and response time (RT) for initial button press. 
Table 2 Diagnostic, demographic, self-report, behavioral, and neuroimaging assessments

\begin{tabular}{|c|c|}
\hline \multicolumn{2}{|c|}{ Diagnostic and demographic assessment } \\
\hline Diagnosis & MINI 6.0 or 7.0 [40] \\
\hline History & Assessment of medical and medication history \\
\hline Treatment completion & Intent to complete treatment form \\
\hline History & Tulsa Life chart interview (see Additional file 1) \\
\hline \multicolumn{2}{|l|}{ Standard self-report scales } \\
\hline Negative valence & $\begin{array}{l}\text { Symptoms of Depression Questionnaire } \\
\text { (SDQ) [41] }\end{array}$ \\
\hline Negative valence & $\begin{array}{l}\text { Overall Anxiety Severity and Impairment } \\
\text { Scale (OASIS) [35] }\end{array}$ \\
\hline Negative valence & State-Trait Anxiety Inventory (STAI) [42] \\
\hline Negative valence & Anxiety Sensitive Index (ASI-3) [43] \\
\hline Negative valence & $\begin{array}{l}\text { Generalized Anxiety Disorder-7 item } \\
\text { (GAD-7) [44] }\end{array}$ \\
\hline Negative valence & Intolerance of Uncertainty Scale (IUS) [45] \\
\hline Negative valence & Penn State Worry Questionnaire [33] \\
\hline Negative valence & Liebowitz Social Anxiety Scale (LSAS) [46] \\
\hline Negative valence & Panic Disorder Severity Scale (PDSS) [47] \\
\hline Negative valence & Beck Depression Inventory-II (BDI-II) [32] \\
\hline Negative valence & Patient Health Questionnaire-9 [48] \\
\hline Negative valence & $\begin{array}{l}\text { Behavioral Activation for Depression } \\
\text { Scale (BADS) [49] }\end{array}$ \\
\hline Substance use & $\begin{array}{l}\text { Customary Drinking and Drug Use Record } \\
\text { (CDDR) [50] }\end{array}$ \\
\hline Trauma & Traumatic Events Questionnaire (TEQ) [51] \\
\hline Trauma & Child Trauma Questionnaire (CTQ) [52] \\
\hline $\begin{array}{l}\text { Positive/negative } \\
\text { valence }\end{array}$ & $\begin{array}{l}\text { Positive and Negative Affect Schedule } \\
\text { (PANAS) [53] }\end{array}$ \\
\hline $\begin{array}{l}\text { Positive/negative } \\
\text { valence }\end{array}$ & $\begin{array}{l}\text { Behavioral Inhibition System/Behavioral } \\
\text { Approach Scale (BIS/BAS) [54] }\end{array}$ \\
\hline $\begin{array}{l}\text { Comorbid anxiety } \\
\text { symptoms }\end{array}$ & $\begin{array}{l}\text { Padua Inventory of Obsessive-Compulsive } \\
\text { Symptoms (PI) [55] }\end{array}$ \\
\hline Personality & Big Five Inventory (BFI) [56] \\
\hline Arousal/interoception & $\begin{array}{l}\text { Multidimensional Assessment of Interoceptive } \\
\text { Awareness (MAIA) [57] }\end{array}$ \\
\hline Sleep & Pittsburgh Sleep Quality Index (PSQI) [58] \\
\hline Physical activity & $\begin{array}{l}\text { International Physical Activity Questionnaire } \\
\text { (IPAQ) [59] }\end{array}$ \\
\hline Disability & Sheehan Disability Scale (SDS) [30] \\
\hline Therapy expectancies & $\begin{array}{l}\text { Credibility/Expectancy Questionnaire } \\
\text { (CEQ) [60] }\end{array}$ \\
\hline Therapy compliance & Homework Rating Scale (HRS) [61] \\
\hline Therapy process & Working Alliance Inventory (WAI) [62] \\
\hline Therapy dropout & Withdrawn Questionnaire (see Additional file 1) \\
\hline $\begin{array}{l}\text { Pre/post } \\
\text { neuroimaging }\end{array}$ & Karolinska Sleepiness Scale: prescan (KSS) [63] \\
\hline $\begin{array}{l}\text { Pre/post } \\
\text { neuroimaging }\end{array}$ & $\begin{array}{l}\text { Positive and Negative Affect Schedule: } \\
\text { prescan (PANAS) [53] }\end{array}$ \\
\hline
\end{tabular}

Table 2 Diagnostic, demographic, self-report, behavioral, and neuroimaging assessments (Continued)

\begin{tabular}{|c|c|}
\hline Negative valence & PROMIS Anxiety \\
\hline Negative valence & PROMIS Depression \\
\hline Sleep & PROMIS Sleep Disturbance \\
\hline Sleep & PROMIS Sleep-Related Impairment \\
\hline Social & PROMIS Emotional Support \\
\hline Social & PROMIS Information Support \\
\hline Social & PROMIS Instrumental Support \\
\hline Social & PROMIS Social Isolation \\
\hline Sex & PROMIS Global Satisfaction with Sex Life \\
\hline Sex & PROMIS Interest in Sex Activity \\
\hline Nicotine & Nicotine dependence \\
\hline Negative affect-anger & NIH Toolbox Anger-Affect Survey \\
\hline Negative affect-anger & NIH Toolbox Anger-Hostility Survey \\
\hline Negative affect-anger & NIH Toolbox Anger-Physical Aggression Survey \\
\hline Negative affect-fear & NIH Toolbox Fear-Affect Survey \\
\hline Negative affect-fear & NIH Toolbox Fear-Somatic Arousal Survey \\
\hline $\begin{array}{l}\text { Psychological well- } \\
\text { being }\end{array}$ & NIH Toolbox General Life Satisfaction Survey \\
\hline $\begin{array}{l}\text { Psychological well- } \\
\text { being }\end{array}$ & NIH Toolbox Meaning and Purpose Survey \\
\hline $\begin{array}{l}\text { Psychological well- } \\
\text { being }\end{array}$ & NIH Toolbox Positive Affect Survey \\
\hline Social & NIH Toolbox Friendship Survey \\
\hline Social & NIH Toolbox Loneliness Survey \\
\hline Stress and self-efficacy & NIH Toolbox Perceived Stress Survey \\
\hline Stress and self-efficacy & NIH Toolbox Self-Efficacy Survey \\
\hline \multicolumn{2}{|c|}{$\begin{array}{l}\text { Behavioral and neuroimaging tasks (see Additional file } 1 \text { for further } \\
\text { description) }\end{array}$} \\
\hline Approach/avoidance & Implicit approach/avoidance task \\
\hline Approach/avoidance & Attentional bias/dot probe task [66] \\
\hline Approach/avoidance & Signal detection reinforcement task \\
\hline Approach/avoidance & Human behavioral pattern monitor (hBPM) \\
\hline Estimated IQ & Wide Range Achievement Test (WRAT) [67] \\
\hline Neuropsychological & $\begin{array}{l}\text { Delis-Kaplan Executive Function System (DKEFS) } \\
\text { color-word test [68] }\end{array}$ \\
\hline Neuropsychological & DKEFS verbal fluency [68] \\
\hline Neuropsychological & $\begin{array}{l}\text { Wechsler Adult Intelligence Scale (WAIS-IV) digit } \\
\text { span [69] }\end{array}$ \\
\hline Neuropsychological & WAIS-IV digit symbol coding [69] \\
\hline Neuropsychological & Finger Tapping Test \\
\hline Neuropsychological & California Verbal Learning Test (CVLT) [70] \\
\hline Neuroimaging & MRI anatomical scan (T1-weighted) \\
\hline Neuroimaging & fMRI resting state with eyes open \\
\hline Neuroimaging & Approach-avoidance conflict (AAC) task \\
\hline Neuroimaging & Emotional faces task (EFT) \\
\hline Neuroimaging & Monetary incentive delay (MID) task \\
\hline
\end{tabular}

NIH PROMIS ${ }^{\oplus}[64]$ and Toolbox ${ }^{\oplus}[65]$ measures 


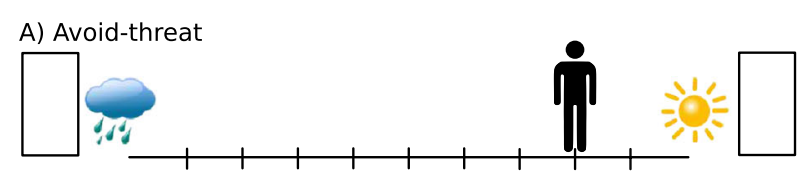

B) Approach-reward

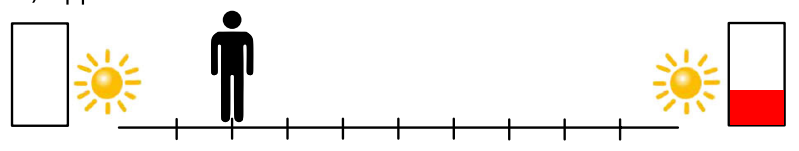

C) Conflict: 2-Point

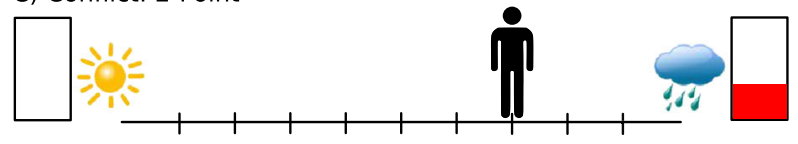

D) Conflict: 4-Point

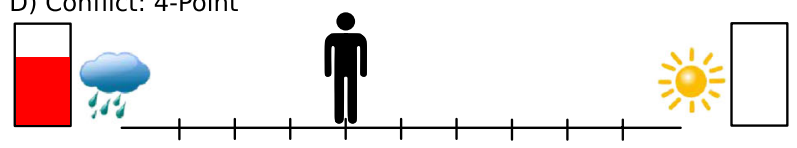

E) Conflict: 6-Point

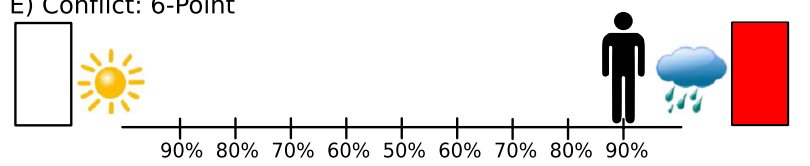

Percent chance of the closest potential outcome occuring if avatar ends at each specific position.

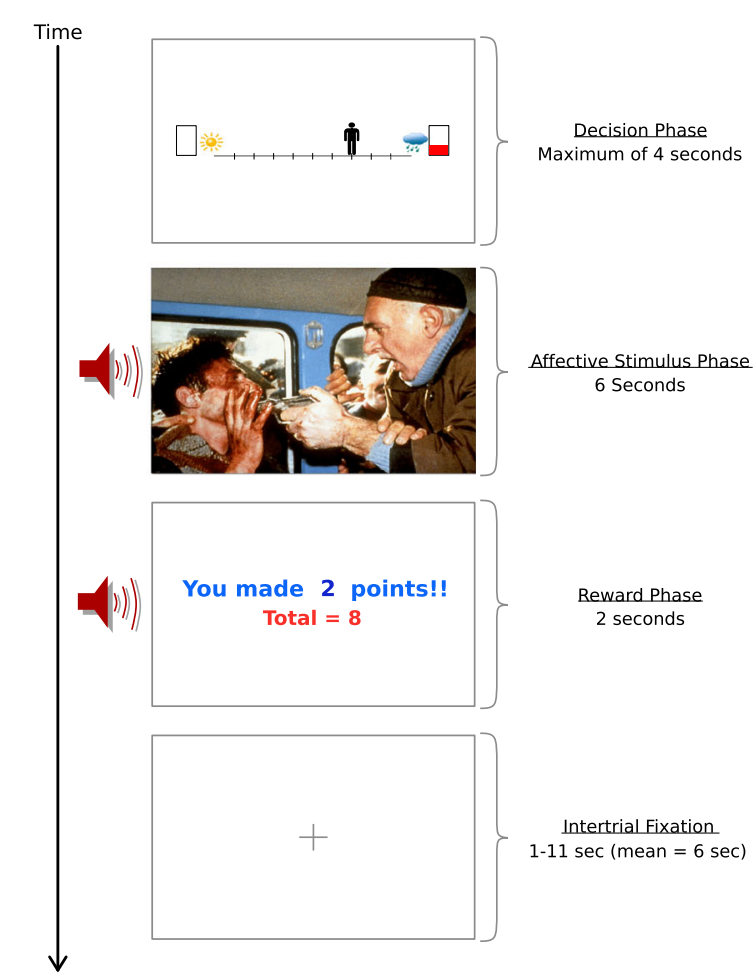

Fig. 3 Approach-avoidance conflict (AAC) task. This figure displays (1) example decision screens displayed during the task for each of the five conditions: avoid-threat, approach-reward, and conflict with 2, 4, or 6 cents offered and (2) the sequence of screens presented for each AAC trial, including a decision phase followed by presentation of the affective image and sound pair (e.g., from International Affective Picture System and International Affective Digitized Sounds system [72, 73]), display of the number of cents received, and then a fixation cross until the next trial begins

During behavioral tasks, the BioPac MP150 system and AcqKnowledge software (BIOPAC Systems, Inc., Goleta, CA, USA) are used to collect galvanic skin conductance, heart rate (electrocardiogram), and respiration rate (respiration transducer). MRI data are collected on a GE MR750 3.0T MRI scanner (GE Healthcare Life Sciences, Chicago, IL, USA). Analysis of Functional NeuroImages [74] is used for processing of MRI data. Electroencephalography (EEG) is simultaneously performed during MRI scanning using a 31-electrode cap attached to an MRI-compatible BrainAmp MR Plus amplifier (Brain Products GmbH, Gilching, Germany). Blood samples for plasma, serum, and peripheral blood mononuclear cells are collected at baseline and posttreatment to quantify biomarkers for future exploratory analyses. See Additional file 1 for further description of MRI, EEG, and blood biomarker methods.

\section{Analysis procedures}

The characteristics of all measures will be examined for missing data and deviation from normality prior to subsequent analyses. Baseline demographic characteristics and attrition data will be contrasted between treatment groups, and analyses will be adjusted to account for potential confounders. For the first aim, we will test the hypotheses that approach and conflict arbitration behavior, and neural responses will explain significant variance in baseline symptoms above and beyond avoidancerelated behavior and neural responses. Approach and avoidance behavior are defined by AAT bias scores; conflict arbitration is defined by RT during AAC conflict trials. For brain responses, we will focus on the AAC task and extracted percentage signal change from the following a priori regions of interest:

1. Approach: left caudate (reward versus no-reward outcome)

2. Avoidance: right amygdala (negative versus positive affective outcome)

3. Conflict: right dIPFC (conflict versus nonconflict decisions)

We will use Huber robust regression with baseline GAD-7 as the dependent variable (DV) and approach, avoidance, and conflict measures as independent variables (IVs).

For the second aim, we will test the hypotheses that approach-related and conflict arbitration behavior and 
neural responses will predict treatment response above and beyond avoidance-related behavior and neural responses. We will use linear mixed models (LMEs) with random subject-level intercepts and slopes, GAD-7 scores across the ten sessions as DVs; baseline GAD-7 as a covariate; and approach, avoidance, and conflict measures as IVs. The main effect of intervention type and its interaction with IVs will be included to determine treatment main effects and moderating effects. We will determine the best set of IVs using the Lasso method [75], and we will use functional linear models [76] to model on-parametric symptom trajectories as needed.

For the third aim, we will test the hypothesis that the degree to which conflict arbitration abilities increase with treatment will positively relate to functional improvement from pre- to posttreatment. We will use LMEs to test main and interaction effects between intervention type and change in AAC conflict arbitration in predicting trajectories of GAD-7 scores over the ten sessions. We will employ the asymmetric distribution of product of coefficients test (versus Baron and Kenny methods) due to the greater power and more appropriate type I error rate it affords [77].

In addition, we are collecting data from other measures for exploratory analyses. For such analyses, we will explore (1) data reduction methods to derive multilevel factors associated with approach, avoidance, and conflict arbitration processes; and (2) use of random forest machine learning, which is particularly appropriate with a large ratio of predictors to participants [78], to identify predictors of treatment outcome.

\section{Sample size and power analysis}

Previous research suggests large effects for fMRI predictors (i.e., $r=0.60-0.75)$ and medium to large effects for behavioral predictors $(r=0.30-0.47)$ of intervention outcomes $[79,80]$. For this study, we aim to recruit 100 participants, which with $20 \%$ attrition would allow for complete longitudinal data for 80 participants (i.e., 40/ intervention). LMEs will include all participants with any postbaseline assessments. Thus, we anticipate approximately 50 participants per intervention for aims $2 / 3$. For relationships between individual predictors and DVs, we estimate having $80 \%$ power to detect medium to large effects $(r=0.27$ for $N=100 ; r=0.37$ for $N=50 ; \alpha=0.05)$. In a model with three predictors (approach, avoidance, conflict arbitration), we also estimate having $80 \%$ power to detect medium to large effects $\left(\eta^{2}=0.11\right.$ for $N=100 ; \eta^{2}=$ 0.24 for $N=50$ ).

\section{Design considerations}

We considered an alternative design where we examined predictors of EXP response compared with an attentional control intervention. We instead decided to identify unique predictors of two theoretically divergent behavioral therapies because (1) the current protocol was not meant to test intervention efficacy compared with "placebo" (because efficacy has been established in previous research); (2) variability in outcomes would be greatest for efficacious, as opposed to placebo, interventions, thus enhancing statistical power for prediction; and (3) identifying unique predictors for two therapies would be most clinically meaningful. We also considered using more unified cognitive behavioral interventions [81]. However, interventions that simultaneously target multiple processes (e.g., both cognitive and behavioral strategies) would have made it more difficult to identify predictors relating to the specific therapeutic target. The goal here is to foster individualized, precision targeting of treatments rather than to apply more broadly targeted treatments to all patients.

\section{Ethics}

Study approval was obtained from the Western Institutional Review Board (WIRB) (protocol 20151232) Additional file 3. Any protocol modifications will be made to records at ClinicalTrials.gov and communicated to study investigators, WIRB, and funding organizations as required.

\section{Gender/minority/pediatric inclusion for research}

Planned study enrollment is reflective of Tulsa County population demographics and is described further in Additional file 1. We will not exclude subjects based on sex, gender, race, or ethnicity. Children are not included, owing to our initial focus on understanding neurobehavioral predictors of exposure therapy for adults with GAD. The variability introduced by developmental changes could reduce sensitivity to detect hypothesized relationships.

\section{Safety}

The risk for adverse events is minimal. The study is a clinical trial but is not a phase III trial, involves only one site, is not blinded, and does not employ high-risk interventions or vulnerable populations. The interventions employed are known to be efficacious for the treatment of anxiety or depression. The PI (RLA), in collaboration with mentor and LIBR scientific director (MPP), are performing the monitoring function for the study. Any unanticipated adverse events will be reported immediately to the LIBR Human Protection Administrator and to the Western IRB. Any adverse events will be included in the annual IRB report.

Each week prior to session, participants complete questionnaires assessing symptom severity and suicidal ideation. As needed, a therapist meets with the participant individually to assess risk and provide referrals or identify emergency services. LIBR is situated on a campus with an inpatient psychiatric hospital (Laureate Psychiatric Clinic 
and Hospital), which has a 24-h clinical assessment department.

\section{Dissemination}

Results will be shared with the scientific and health professional community through presentation at national and international scientific meetings and publication in scientific journals. The full protocol and statistical code used for data analysis will be provided with resulting publications. To disseminate results to the general public, all final peer-reviewed manuscripts will be submitted to the PubMed Central digital archive in compliance with the NIH public access policy. The PI will maintain a local website where lay summaries of scientific results will be provided, with appropriate links to scientific presentations and publications. The PI will ensure that the summary of results information is submitted to ClinicalTrials.gov.

\section{Discussion}

To enhance treatment effectiveness and efficiency for individuals with anxiety and depression, it will be beneficial to understand why many patients do not respond optimally to gold standard therapies and to be able to predict, before treatment begins which patients will respond to which treatments. The study detailed in this protocol article represents a first step toward these goals, using the RDoC framework [12] to probe multilevel predictors of EXP versus BA therapy for GAD. This work addresses the NIMH strategic plan by (1) integrating biological markers and behavioral indicators associated with GAD (strategy 1.3) and (2) using a multidimensional design to ascertain individual predictors of therapy response (strategy 3.1) that will (3) inform future research developing strategies for personalized mental health care (strategy 3.2).

Czajkowski et al. [82] presented the Obesity-Related Behavioral Intervention Trials (ORBIT) model as a strategy for the development of novel behavioral treatments. Although the ORBIT model was developed from a health psychology perspective, it is also relevant more broadly. These authors proposed that "the hypothesis that change in a behavioral risk factor could solve a clinical problem is one of the entry points for behavioral treatment development" [82]. Similarly, the identification of neural and behavioral risk factors for response or nonresponse to different psychosocial treatments could provide entry points for development of novel, personalized mental health treatment strategies. If specific behaviors (i.e., conflict arbitration difficulties, approach motivation) and/or neural networks (e.g., dlPFC, striatum) can be shown to predict therapy outcomes, research could then turn toward identifying strategies for modifying these factors. This could involve neuromodulation approaches (e.g., fMRI real-time neurofeedback, transcranial magnetic stimulation) [83], cognitive or behavioral strategies (e.g., cognitive control or attention bias training, cognitive rehabilitation strategies $[84,85])$, or pharmacologic approaches (e.g., dopaminergic or $\mathrm{N}$ methyl-D-aspartate-related drugs to target motivational or cognitive circuitry, respectively) [86]. This approach is in concert with NIMH's more recent experimental therapeutic approach to clinical trials.

Strengths of the described protocol include the randomization of participants to two interventions, both of which have documented efficacy but target different and specific processes rooted in distinct neural circuitry. The study is strengthened by inclusion of multilevel assessments-self-report, behavioral, and neurobiological-to probe domains of positive and negative valence and cognitive control. In addition, the domains and interventions assessed are relevant transdiagnostically. Thus, if promising results are identified, future studies could use similar protocols to test whether findings can be generalized to other anxiety disorder and depressive disorder populations. In addition, the interventions are manualized and identical in regard to format, frequency, duration, and level of therapist training, and we use consultation with experts in each.

The study is not without limitations. Although our target sample size is larger than any published fMRI study predicting GAD treatment response, the sample size is underpowered to detect small effect sizes or for independent replications. Thus, results identified from the current study will require follow-up replication. Also, the trial is being conducted at only one site, so generalizability across sites would need to be determined in future research. The delivery of the intervention in a group format allows for greater control and balance regarding which therapists are providing the treatment, and it increases cost and time efficiency of the trial. However, this may limit the generalizability of findings to individual therapy.

This protocol provides a framework for how studies may be designed to move the field toward neuroscienceinformed and personalized psychosocial treatments. The results of the trial will have implications for approachavoidance processing in GAD; relationships between levels of analysis (i.e., behavioral, neural); and, most important, predictors of behavioral therapy outcome. The results also have the potential to inform a line of research aimed at optimizing psychosocial treatment for anxiety and depressive disorders from a holistic, neuroscience, and behaviorally informed perspective and to move us closer to truly personalized precision approaches to psychiatric treatment.

\section{Trial status}

Study approval was obtained from the Western Institutional Review Board (WIRB; protocol 20151232). Recruitment began on June 7, 2016, and the approximate 
date when recruitment will be completed is April 1, 2021. The study was retrospectively registered within 21 days of first participant enrollment in accordance with FDAAA 801 (ClinicalTrials.gov identifier NCT02807480; registration date June 21, 2016).

\section{Supplementary information}

Supplementary information accompanies this paper at https://doi.org/10. 1186/s13063-019-3802-9.

Additional file 1. Supplementary material: Additional information on methods and analysis of the interventions, assessments (behavioral and neuroimaging), blood biomarker storage, and plans for enrollment.

Additional file 2. SPIRIT checklist: Information regarding the recommended items for a clinical trial protocol paper.

Additional file 3. Approval letter from Western Institutional Review Board.

Additional file 4. K23 Notice of Award: Letter from the National Institute of Mental Health informing the principal investigator of the award granted for this protocol.

Additional file 5. Study consent form: The informed consent document approved by the Western Institutional Review Board.

\section{Abbreviations}

AAC: Approach-avoidance conflict; AAT: Approach-Avoidance Task: ABC: Antecedent, behavior, and consequence; ACTION: Assess behavior/ mood, choose alternate responses, try out alternate responses, integrate these alternatives, observe results, now evaluate; BA: Behavioral activation; BADS-SF: Behavioral Activation for Depression Scale-Short Form; BDI-II: Beck Depression Inventory II; BDI-II SI: Beck Depression Inventory suicidal ideation item; CBT: Cognitive behavioral therapy; CEQ: Credibility/Expectancy Questionnaire; dIPFC: Dorsolateral prefrontal cortex; DSM-5: Diagnostic and Statistical Manual of Mental Disorders, Fifth Edition; DV: Dependent variable; EEG: Electroencephalography; EXP: Exposure therapy; fMRI: Functional magnetic resonance imaging; GAD: Generalized anxiety disorder; GAD7: Generalized Anxiety Disorder 7-item scale; HRS: Homework Rating Scale; IV: Independent variable; LIBR: Laureate Institute for Brain Research; LME: Linear mixed models; LSAS: Liebowitz Social Anxiety Scale; MDD: Major depressive disorder; MINI: Mini International Neuropsychiatric Interview; MRI: Magnetic resonance imaging; $\mathrm{NIH}$ : National Institutes of Health; NIMH: National Institute of Mental Health; OASIS: Overall Anxiety Severity and Impairment Scale; ORBIT: Obesity-Related Behavioral Intervention Trials; PDSS: Panic Disorder Severity Scale; PFC: Prefrontal cortex; PROMIS Anx \& Dep: Patient-Reported Outcomes Measurement Information System anxiety and depression scales; PSC: Percentage signal change; PSWQ: Penn State Worry Questionnaire; RDoC: Research Domain Criteria; REDCap: Research Electronic Data Capture; RT: Response time; SDS: Sheehan Disability Scale; SPIRIT: Standard Protocol Items: Recommendations for Interventional Trials; SSRI: Selective serotonin reuptake inhibitor; TRAC: trigger, response, alternative coping; TRAP: trigger, response, avoidance pattern; Tx: treatment; WAI: Working Alliance Inventory; WIRB: Western Institutional Review Board

\section{Acknowledgements}

We acknowledge the support and work of graduate students and postdoctoral fellows who have or will contribute to the delivery of the interventions described in this protocol. In addition, we acknowledge Catherine Ayers, PhD, for providing the group-based anxiety treatment manual used in her previous work to inform the exposure-based manual used in the current protocol. Last, we also acknowledge the participants who have or will in the future volunteer their time to this study.

\section{Authors' contributions}

JS contributed to data collection, literature search, intervention delivery, creation of figures/tables, and initial drafting and revision of the manuscript. EA contributed to data collection, literature search, creation of figures/tables, and revision of the manuscript. NK contributed to literature search, intervention delivery, and revision of the manuscript. ANC contributed to study design, data collection, intervention delivery, revision of intervention manuals, and revision of the manuscript. KTC contributed to data collection, literature search, and revision of the manuscript. TJM contributed to data collection, literature search, and revision of the manuscript. BM contributed to data collection, literature search, creation of figures/tables, and revision of the manuscript. MPM contributed to study design, analytic plan, and revision of the manuscript. MGC contributed to study design, analytic plan, exposure therapy manual development, and revision of the manuscript. JB contributed to study design, analytic plan, and revision of the manuscript. JA contributed to study design, analytic plan, and revision of the manuscript. CM contributed to behavioral activation manual development, consultation on intervention delivery, and revision of the manuscript. KWT provided revision of the exposure therapy manual, consultation on intervention delivery, and revision of the manuscript. WKT contributed to the analytic plan and revision of the manuscript. RLA contributed to study design, data collection, intervention manual development and delivery, supervision of intervention delivery, data analysis, literature search, creation of figures/tables, and initial drafting and revision of the manuscript. All authors read and approved the final manuscript.

\section{Funding}

This work was supported by National Institute of Mental Health grant K23MH108707 (Robin L. Aupperle, PhD, principal investigator) and the William K. Warren Foundation (Additional file 4). The study sponsor and funding organizations will have no direct role in data collection, analysis, or interpretation; trial design; patient recruitment; or any aspect pertinent to the study. The authors were not paid to write this article by a pharmaceutical company or other agency. The authors will have full access to all the data in the study and have final responsibility for the decision to submit it for publication.

\section{Availability of data and materials}

The datasets analyzed during the current study are available from the corresponding author on reasonable request.

\section{Ethics approval and consent to participate}

Study approval was obtained under WIRB protocol 20151232. Informed consent will be obtained from all study participants. Any protocol modifications will be made to records on ClinicalTrials.gov and communicated to study investigators, WIRB, and funding organizations as required.

\section{Consent for publication}

Not applicable. Individually identifiable data is not included in this manuscript.

\section{Competing interests}

Jessica Santiago, Elisabeth Akeman, Kelly Cosgrove, Bailey Mathis, Timothy McDermott, and Drs. James Abelson and Wes Thompson have no conflicts of interest to declare. Drs. Namik Kirlic and Jerzy Bodurka report receiving grants from the National Institute for General Medical Sciences. Dr. Ashley Clausen is funded by the Department of Veterans Affairs Office of Academic Affiliations Advanced Fellowship Program in Mental IIIness Research and Treatment; the Medical Research Service of the Durham VA Health Care System; and the Department of Veterans Affairs Mid-Atlantic Mental Illness Research, Education, and Clinical Center (MIRECC). Dr. Michelle Craske reports receiving grants from the National Institute of Mental Health. Dr. Christopher Martell reports receiving royalties for four books on the topic of behavioral activation. Dr. Kathryn Wolitzky-Taylor reports receiving grants from the National Institute on Alcohol Abuse and Alcoholism and the National Institute on Drug Abuse. Dr. Martin Paulus has received royalties for an article about methamphetamine published in UpToDate and grants from the National Institute of General Medical Sciences and the National Institute of Mental Health. Dr. Robin Aupperle reports receiving grants from the National Institute of Mental Health, the National Institute of General Medical Sciences, and Oklahoma Science and Technology Research and Development. The views expressed in this article are those of the authors and do not necessarily reflect the position or policy of the Department of Veterans Affairs or the U.S. government. 


\section{Author details}

'Laureate Institute for Brain Research, 6655 South Yale Avenue, Tulsa, OK 74136, USA. ${ }^{2}$ School of Community Medicine, University of Tulsa, Tulsa, OK, USA. ${ }^{3}$ Department of Psychology, University of Tulsa, Tulsa, OK, USA. ${ }^{4}$ VA Mid-Atlantic Mental Illness Research, Education and Clinical Center, Durham, NC, USA. ${ }^{5}$ Duke University Brain Imaging and Analysis Center, Durham, NC, USA. ${ }^{6}$ Psychology, Psychiatry and Biobehavioral Sciences, University of California, Los Angeles, Los Angeles, CA, USA. Department of Psychiatry, University of Michigan, Ann Arbor, MI, USA. ${ }^{8}$ Department of Psychological and Brain Sciences, University of Massachusetts-Amherst, Amherst, MA, USA. ${ }^{9}$ Stephenson School of Biomedical Engineering, The University of Oklahoma, Norman, OK, USA. ${ }^{10}$ Family Medicine and Public Health, University of California, San Diego, San Diego, CA, USA.

\section{Received: 13 August 2019 Accepted: 11 October 2019} Published online: 06 January 2020

\section{References}

1. Kessler RC. The costs of depression. Psychiatr Clin North Am. 2012;35(1):114. https://doi.org/10.1016/j.psc.2011.11.005.

2. Wittchen HU. Generalized anxiety disorder: prevalence, burden, and cost to society. Depress Anxiety. 2002;16(4):162-71. https:/doi.org/10.1002/da.10065.

3. Hoffman DL, Dukes EM, Wittchen HU. Human and economic burden of generalized anxiety disorder. Depress Anxiety. 2008;25(1):72-90. https://doi. org/10.1002/da.20257.

4. Penninx BW, Nolen WA, Lamers F, Zitman FG, Smit JH, Spinhoven P, et al. Two-year course of depressive and anxiety disorders: results from the Netherlands Study of Depression and Anxiety (NESDA). J Affect Disord. 2011;133(1-2):76-85. https://doi.org/10.1016/j.jad.2011.03.027.

5. Moffitt TE, Harrington H, Caspi A, Kim-Cohen J, Goldberg D, Gregory AM, et al. Depression and generalized anxiety disorder: cumulative and sequential comorbidity in a birth cohort followed prospectively to age 32 years. Arch Gen Psychiatry. 2007;64(6):651-60. https://doi.org/10.1001/archpsyc.64.6.651.

6. Sherbourne CD, Wells KB. Course of depression in patients with comorbid anxiety disorders. J Affect Disord. 1997;43(3):245-50. https://doi.org/10.1016/ s0165-0327(97)01442-0.

7. Hidalgo RB, Tupler LA, Davidson JR. An effect-size analysis of pharmacologic treatments for generalized anxiety disorder. J Psychopharmacol. 2007;21(8): 864-72. https://doi.org/10.1177/0269881107076996.

8. Hofmann SG, Smits JAJ. Cognitive-behavioral therapy for adult anxiety disorders: a meta-analysis of randomized placebo-controlled trials. J Clin Psychiatry. 2008;69(4):621-32. https://doi.org/10.4088/jcp.v69n0415.

9. Hollon SD, Stewart MO, Strunk D. Enduring effects for cognitive behavior therapy in the treatment of depression and anxiety. Annu Rev Psychol. 2006;57:285-315. https://doi.org/10.1146/annurev.psych.57.102904.190044.

10. Cuijpers P, Sijbrandij M, Koole S, Huibers M, Berking M, Andersson G. Psychological treatment of generalized anxiety disorder: a meta-analysis. Clin Psychol Rev. 2014;34(2):130-40. https://doi.org/10.1016/j.cpr.2014.01.002.

11. Marciniak MD, Lage MJ, Dunayevich E, Russell JM, Bowman L, Landbloom RP, et al. The cost of treating anxiety: the medical and demographic correlates that impact total medical costs. Depress Anxiety. 2005;21(4):178-84.

12. Sanislow CA, Pine DS, Quinn KJ, Kozak MJ, Garvey MA, Heinssen RK, et al. Developing constructs for psychopathology research: research domain criteria. J Abnorm Psychol. 2010;1 19(4):631-9. https://doi.org/10.1037/a0020909.

13. Craske MG. Honoring the past, envisioning the future: $A B C T$ 's 50 th anniversary presidential address. Behav Ther. 2018;49(2):151-64. https://doi. org/10.1016/j.beth.2017.05.003.

14. Fernandes BS, Williams LM, Steiner J, Leboyer M, Carvalho AF, Berk M. The new field of 'precision psychiatry'. BMC Med. 2017;15:80.

15. Altamura AC, Dell'osso B, D'Urso N, Russo M, Fumagalli S, Mundo E. Duration of untreated illness as a predictor of treatment response and clinical course in generalized anxiety disorder. CNS Spectr. 2008;13(5):415-22.

16. Blair KS, Blair R. A cognitive neuroscience approach to generalized anxiety disorder and social phobia. Emot Rev. 2012;4(2):133-8. https://doi.org/10. $1177 / 1754073911430251$

17. Santos VA, Carvalho DD, Van Ameringen M, Nardi AE, Freire RC. Neuroimaging findings as predictors of treatment outcome of psychotherapy in anxiety disorders. Prog Neuropsychopharmacol Biol Psychiatry. 2019;91:60-71. https://doi.org/10.1016/j.pnpbp.2018.04.001.

18. Ball TM, Stein MB, Ramsawh HJ, Campbell-Sills L, Paulus MP. Single-subject anxiety treatment outcome prediction using functional neuroimaging.
Neuropsychopharmacology. 2014;39(5):1254-61. https://doi.org/10.1038/ npp.2013.328.

19. Behar E, DiMarco ID, Hekler EB, Mohlman J, Staples AM. Current theoretical models of generalized anxiety disorder (GAD): conceptual review and treatment implications. J Anxiety Disord. 2009;23(8):1011-23. https://doi.org/ 10.1016/j.janxdis.2009.07.006.

20. Aupperle RL, Paulus MP. Neural systems underlying approach and avoidance in anxiety disorders. Dialogues Clin Neurosci. 2010;12(4):517-31.

21. Millan MJ. The neurobiology and control of anxious states. Prog Neurobiol. 2003;70(2):83-244

22. Kirlic N, Young J, Aupperle RL. Animal to human translational paradigms relevant for approach avoidance conflict decision making. Behav Res Ther. 2017;96:14-29. https://doi.org/10.1016/j.brat.2017.04.010.

23. Aupperle RL, Melrose AJ, Francisco A, Paulus MP, Stein MB. Neural substrates of approach-avoidance conflict decision-making. Hum Brain Mapp. 2015; 36(2):449-62. https://doi.org/10.1002/hbm.22639.

24. Aupperle RL, Sullivan S, Melrose AJ, Paulus MP, Stein MB. A reverse translational approach to quantify approach-avoidance conflict in humans. Behav Brain Res. 2011;225(2):455-63. https://doi.org/10.1016/j.bbr.2011.08.003.

25. Craske MG, Barlow DH. Mastery of your anxiety and worry. 2nd ed. San Antonio: Psychological Corp.; 2006

26. Dillon DG, Rosso IM, Pechtel P, Killgore WD, Rauch SL, Pizzagalli DA. Peril and pleasure: an RDOC-inspired examination of threat responses and reward processing in anxiety and depression. Depress Anxiety. 2014;31(3): 233-49. https://doi.org/10.1002/da.22202.

27. Cuijpers $P$, van Straten A, Warmerdam L. Behavioral activation treatments of depression: a meta-analysis. Clin Psychol Rev. 2007;27(3):318-26. https://doi. org/10.1016/j.cpr.2006.11.001.

28. Craske MG, Roy-Byrne PP, Stein MB, Sullivan G, Sherbourne C, Bystritsky A. Treatment for anxiety disorders: efficacy to effectiveness to implementation. Behav Res Ther. 2009;47(11):931-7. https://doi.org/10.1016/j.brat.2009.07.012.

29. Martell CR, Dimidjian S, Herman-Dunn R. Behavioral activation for depression: a clinician's guide. New York: Guilford Press; 2013.

30. Sheehan DV, Harnett-Sheehan K, Raj BA. The measurement of disability. Int Clin Psychopharmacol. 1996;11 Suppl 3:89-95.

31. Schalet BD, Pilkonis PA, Yu L, Dodds N, Johnston $\mathrm{KL}$, Yount $\mathrm{S}$, et al. Clinical validity of PROMIS depression, anxiety, and anger across diverse clinical samples. J Clin Epidemiol. 2016;73:119-27. https://doi.org/10.1016/j.jclinepi.2015.08.036.

32. Beck AT, Steer RA, Ball R, Ranieri W. Comparison of Beck Depression Inventories-IA and -II in psychiatric outpatients. J Pers Assess. 1996;67(3): 588-97. https://doi.org/10.1207/s15327752jpa6703_13.

33. Meyer TJ, Miller ML, Metzger RL, Borkovec TD. Development and validation of the Penn State Worry Questionnaire. Behav Res Ther. 1990;28(6):487-95. https://doi.org/10.1016/0005-7967(90)90135-6.

34. American Psychiatric Association. Diagnostic and statistical manual of mental disorders (DSM-5 ${ }^{\circledR}$ ). 5th ed. Washington, DC: American Psychiatric Publishing; 2013.

35. Norman SB, Hami Cissell S, Means-Christensen AJ, Stein MB. Development and validation of an overall anxiety severity and impairment scale (OASIS). Depress Anxiety. 2006;23(4):245-9.

36. Dimidjian S, Hollon SD, Dobson KS, Schmaling KB, Kohlenberg RJ, Addis ME, et al. Randomized trial of behavioral activation, cognitive therapy, and antidepressant medication in the acute treatment of adults with major depression. J Consult Clin Psychol. 2006;74(4):658-70. https://doi.org/10.1037/0022-006X.74.4.658.

37. Arch JJ, Ayers CR, Baker A, Almklov E, Dean DJ, Craske MG. Randomized clinical trial of adapted mindfulness-based stress reduction versus group cognitive behavioral therapy for heterogeneous anxiety disorders. Behav Res Ther. 2013;51(4-5):185-96. https://doi.org/10.1016/j.brat.2013.01.003.

38. Craske MG, Treanor M, Conway CC, Zbozinek T, Vervliet B. Maximizing exposure therapy: an inhibitory learning approach. Behav Res Ther. 2014;58: 10-23. https://doi.org/10.1016/j.brat.2014.04.006.

39. Harris PA, Taylor R, Thielke R, Payne J, Gonzalez N, Conde JG. Research Electronic Data Capture (REDCap) - a metadata-driven methodology and workflow process for providing translational research informatics support. J Biomed Inform. 2009;42(2):377-81. https://doi.org/10.1016/j.jbi.2008.08.010.

40. Sheehan DV, Lecrubier $Y$, Sheehan KH, Amorim P, Janavs J, Weiller E, et al. The Mini-International Neuropsychiatric Interview (M.I.N.I.): development and validation of a structured diagnostic psychiatric interview. J Clin Psychiatry. 1998; 59 Suppl 20:22-33.

41. Pedrelli P, Blais MA, Alpert JE, Shelton RC, Walker RS, Fava M. Reliability and validity of the Symptoms of Depression Questionnaire (SDQ). CNS Spectr. 2014;19(6):535-46. https://doi.org/10.1017/S1092852914000406. 
42. Spielberger C. State-Trait Anxiety Inventory (Form Y) manual. Palo Alto: Mind Garden; 1983.

43. Taylor S, Koch WJ, McNally RJ, Crockett DJ. Conceptualizations of anxiety sensitivity. Psychol Assess. 1992;4(2):245.

44. Spitzer RL, Kroenke K, Williams JB, Lowe B. A brief measure for assessing generalized anxiety disorder: the GAD-7. Arch Intern Med. 2006;166(10): 1092-7. https://doi.org/10.1001/archinte.166.10.1092.

45. Buhr K, Dugas MJ. The intolerance of uncertainty scale: psychometric properties of the English version. Behav Res Ther. 2002;40(8):931-45. https:// doi.org/10.1016/s0005-7967(01)00092-4.

46. Baker SL, Heinrichs N, Kim HJ, Hofmann SG. The Liebowitz social anxiety scale as a self-report instrument: a preliminary psychometric analysis. Behav Res Ther. 2002:40(6):701-15.

47. Shear MK, Rucci P, Williams J, Frank E, Grochocinski V, Vander Bilt J, et al. Reliability and validity of the Panic Disorder Severity Scale: replication and extension. J Psychiatr Res. 2001;35(5):293-6. https://doi.org/10.1016/s00223956(01)00028-0.

48. Kroenke K, Spitzer RL, Williams JB. The PHQ-9: validity of a brief depression severity measure. J Gen Intern Med. 2001;16(9):606-13. https://doi.org/10. 1046/j.1525-1497.2001.016009606.x.

49. Kanter JW, Mulick PS, Busch AM, Berlin KS, Martell CR. The Behavioral Activation for Depression Scale (BADS): psychometric properties and factor structure. J Psychopathol Behav. 2007;29(3):191. https://doi.org/10.1007/ s10862-006-9038-5.

50. Brown SA, Myers MG, Lippke L, Tapert SF, Stewart DG, Vik PW. Psychometric evaluation of the Customary Drinking and Drug Use Record (CDDR): a measure of adolescent alcohol and drug involvement. J Stud Alcohol. 1998; 59(4):427-38. https://doi.org/10.15288/jsa.1998.59.427.

51. Vrana S, Lauterbach D. Prevalence of traumatic events and post-traumatic psychological symptoms in a nonclinical sample of college students. J Trauma Stress. 1994;7(2):289-302.

52. Bernstein DP, Fink L, Handelsman L, Foote J, Lovejoy M, Wenzel K, et al. Initial reliability and validity of a new retrospective measure of child abuse and neglect. Am J Psychiatry. 1994;151(8):1132. https://doi.org/10.1176/ajp.151.8.1132.

53. Watson D, Clark LA, Tellegen A. Development and validation of brief measures of positive and negative affect: the PANAS scales. J Pers Soc Psychol. 1988;54(6):1063-70. https://doi.org/10.1037//0022-3514.54.6.1063.

54. Carver CS, White TL. Behavioral inhibition, behavioral activation, and affective responses to impending reward and punishment: the BIS/BAS scales. J Pers Soc Psychol. 1994;67(2):319.

55. Burns GL, Keortge SG, Formea GM, Sternberger LG. Revision of the Padua Inventory of obsessive compulsive disorder symptoms: distinctions between worry, obsessions, and compulsions. Behav Res Ther. 1996;34(2):163-73.

56. John OP, Srivastava S. The Big Five trait taxonomy: history, measurement, and theoretical perspectives. Handbook of personality: Theory and research. 1999;2(1999):102-38

57. Mehling WE, Price C, Daubenmier JJ, Acree M, Bartmess E, Stewart A. The Multidimensional Assessment of Interoceptive Awareness (MAIA). PLoS One. 2012;7(11):e48230. https://doi.org/10.1371/journal.pone.0048230.

58. Carpenter JS, Andrykowski MA. Psychometric evaluation of the Pittsburgh Sleep Quality Index. J Psychosom Res. 1998;45(1):5-13. https://doi.org/10. 1016/s0022-3999(97)00298-5.

59. Craig CL, Marshall AL, Sjöström M, Bauman AE, Booth ML, Ainsworth BE, et al. International Physical Activity Questionnaire: 12-country reliability and validity. Med Sci Sports Exerc. 2003;35(8):1381-95. https://doi.org/10.1249/01. MSS.0000078924.61453.FB.

60. Devilly GJ, Borkovec TD. Psychometric properties of the credibility/ expectancy questionnaire. J Behav Ther Exp Psychiatry. 2000;31(2):73-86.

61. Kazantzis N, Deane FP, Ronan KR. Assessing compliance with homework assignments: review and recommendations for clinical practice. J Clin Psychol. 2004;60(6):627-41. https://doi.org/10.1002/jclp.10239.

62. Hatcher RL, Gillaspy JA. Development and validation of a revised short version of the Working Alliance Inventory. Psychother Res. 2006;16(1):12-25. https://doi.org/10.1080/10503300500352500.

63. Kaida K, Takahashi M, Åkerstedt T, Nakata A, Otsuka Y, Haratani T, et al. Validation of the Karolinska Sleepiness Scale against performance and EEG variables. Clin Neurophysiol. 2006;117(7):1574-81. https://doi.org/10.1016/j. clinph.2006.03.011.

64. Broderick JE, DeWitt EM, Rothrock N, Crane PK, Forrest CB. Advances in patient-reported outcomes: the NIH PROMIS ${ }^{\circledR}$ measures. EGEMS. 2013;1(1): 2015. https://doi.org/10.13063/2327-9214.
65. Salsman JM, Butt Z, Pilkonis PA, Cyranowski JM, Zill N, Hendrie HC, et al. Emotion assessment using the NIH Toolbox. Neurology. 2013:80(11 Supplement 3):S76-86. https://doi.org/10.1212/WNL.0b013e3182872e11.

66. Matsumoto D, Ekman P. Japanese and Caucasian facial expressions of emotion (JACFEE). San Francisco: Intercultural and Emotion Research Laboratory, Department of Psychology, San Francisco State University; 1988.

67. Wilkinson GS, Robertson GJ. Wide Range Achievement Test 4 professional manual. Lutz: Psychological Assessment Resources; 2006.

68. Delis DC, Kaplan E. Delis-Kaplan Executive Function battery. San Antonio: Psychological Corpor.; 2001.

69. Wechsler D, Coalson DL, Raiford SE. WAIS-IV technical and interpretive manual. San Antonio: Psychological Corp.; 2008.

70. Delis DC, Kramer JH, Kaplan E, Ober BA. The California Verbal Learning Test. Second ed. San Antonio: The Psychological Corp.; 2000.

71. Heuer K, Rinck M, Becker ES. Avoidance of emotional facial expressions in social anxiety: the approach-avoidance task. Behav Res Ther. 2007;45(12): 2990-3001. https://doi.org/10.1016/j.brat.2007.08.010.

72. Lang PJ, Bradley MM, Cuthbert BN. International Affective Picture System (IAPS): affective ratings of pictures and instruction manual. Technical Report A-8. Gainesville: University of Florida; 2008.

73. Bradley MM, Lang PJ. International Affective Digitized Sounds (IADS): stimuli, instruction manual, and affective ratings. Tech. Rep. No. B-2. Gainesville: Center for Research in Psychophysiology, University of Florida; 1999.

74. Cox RW. AFNI: software for analysis and visualization of functional magnetic resonance neuroimages. Comput Biomed Res. 1996;29(3):162-73. https:// doi.org/10.1006/cbmr.1996.0014.

75. Tibshirani R. Regression shrinkage and selection via the lasso. J R Stat Soc Series B Stat Methodol. 1996:58(1):267-88.

76. Ramsay J, Silverman BW. Functional data analysis. New York: Springer Science + Business; 2005.

77. Mackinnon DP, Lockwood CM, Hoffman JM, West SG, Sheets V. A comparison of methods to test mediation and other intervening variable effects. Psychol Methods. 2002;7(1):83-104.

78. Bureau A, Dupuis J, Falls K, Lunetta KL, Hayward B, Keith TP, et al. Identifying SNPs predictive of phenotype using random forests. Genet Epidemiol. 2005: 28(2):171-82.

79. Arch JJ, Ayers CR. Which treatment worked better for whom? Moderators of group cognitive behavioral therapy versus adapted mindfulness based stress reduction for anxiety disorders. Behav Res Ther. 2013;51(8):434-42. https://doi.org/10.1016/j.brat.2013.04.004.

80. Nitschke JB, Sarinopoulos I, Oathes DJ, Johnstone T, Whalen PJ, Davidson RJ, et al. Anticipatory activation in the amygdala and anterior cingulate in generalized anxiety disorder and prediction of treatment response. Am J Psychiatry. 2009;166(3):302-10. https://doi.org/10.1176/appi.ajp.2008.07101682.

81. Farchione TJ, Fairholme CP, Ellard KK, Boisseau CL, Thompson-Hollands J, Carl JR, et al. Unified protocol for transdiagnostic treatment of emotional disorders: a randomized controlled trial. Behav Ther. 2012;43(3):666-78. https://doi.org/10.1016/j.beth.2012.01.001.

82. Czajkowski SM, Powell LH, Adler N, Naar-King S, Reynolds KD, Hunter CM, et al. From ideas to efficacy: the ORBIT model for developing behavioral treatments for chronic diseases. Health Psychol. 2015;34(10):971. https://doi. org/10.1037/hea0000161.

83. Young KD, Zotev V, Phillips R, Misaki M, Drevets WC, Bodurka J. Amygdala real-time functional magnetic resonance imaging neurofeedback for major depressive disorder: a review. Psychiatry Clin Neurosci. 2018;72(7):466-81. https://doi.org/10.1111/pcn.12665.

84. Clausen AN, Thelen J, Francisco AJ, Bruce J, Martin L, McDowd J, et al. Computer-based executive function training for combat veterans with PTSD: a pilot clinical trial assessing feasibility and predictors of dropout. Front Psychiatry. 2019;10:62. https://doi.org/10.3389/fpsyt.2019.00062.

85. Amir N, Beard C, Burns M, Bomyea J. Attention modification program in individuals with generalized anxiety disorder. J Abnorm Psychol. 2009; 118(1):28-33. https://doi.org/10.1037/a0012589.

86. Choi DC, Rothbaum BO, Gerardi M, Ressler KJ. Pharmacological enhancement of behavioral therapy: focus on posttraumatic stress disorder. In: Stein M, Steckler T, editors. Behavioral neurobiology of anxiety and its treatment. Vol. 2. Berlin: Springer; 2009. p. 279-99.

\section{Publisher's Note}

Springer Nature remains neutral with regard to jurisdictional claims in published maps and institutional affiliations. 